\title{
Feeling More Pain, Yet Showing Less: The Influence of Social Threat on Pain
}

\author{
Pim A. M. Peeters* and Johan W. S. Vlaeyen ${ }^{*}, \dagger$ \\ * Department of Clinical Psychological Science, Maastricht University, Maastricht, The Netherlands. \\ ${ }^{\dagger}$ Research Group Health Psychology, University of Leuven, Leuven, Belgium.
}

\begin{abstract}
The present study investigated the effects of social threat to physical integrity on reported pain and facial pain expression. Predictions of a cognitive appraisal model and a communicative perspective on pain expression were compared. Participants $(N=67)$ received 5 electric pain stimuli administered by a confederate. They were led to believe that 5 pain stimuli were the minimum, a fixed amount, or the maximum number of pain stimuli allowed, thereby varying the social threat posed by the confederate. Reported pain and facial pain expression were recorded during the delivery of pain stimuli. Increased perceived social threat led to an increase of reported pain, specifically for high pain catastrophizing participants, while it led to a reduction of facial pain expression. This is the first study to demonstrate that a social threat manipulation has opposite effects on reported pain and facial expression, suggesting differences in adaptive function for both forms of pain expression.

Perspective: This is the first demonstration showing an increase in verbal pain report and a decrease in nonverbal pain expression at the same time during social threat. This knowledge may contribute to improving pain assessment in different contexts.
\end{abstract}

(c) 2011 by the American Pain Society

Key words: Social context, social threat, pain expression, facial expression, evolutionary psychology, threat of pain.

T

There is a growing number of studies demonstrating that social context factors, such as the presence or absence of others, influence the reported experience and nonverbal behavior of individuals in pain. Most of these studies have focused on social support, ${ }^{2}$ spouse solicitousness, ${ }^{9}$ or simply the presence of others. ${ }^{18}$ However, individuals in pain may also experience social situations as threatening, either to physical integrity, such as intentionally inflicted pain, or to psychosocial integrity, such as stigmatization or social exclusion. Experimental research on the effects of social threats on the verbal or nonverbal expression of pain is currently lacking, which limits our knowledge of pain expression and its underlying mechanisms.

Based on a cognitive appraisal model of pain, ${ }^{17,20}$ social safety cuing ${ }^{23}$ is 1 mechanism that may underlie

Received June 23, 2011; Revised July 19, 2011; Accepted July 22, 2011. Supported by the NWO Social Sciences Research Council of the Netherlands, Grant Nos. 021-002-039 and 453-04-003 and an Odysseus Grant by the Fund for Scientific Research-Flanders, Belgium (FWO).

The authors have no conflicts of interest to report.

Address reprint requests to Pim A. M. Peeters, MSC, Department of Clinical Psychological Science, Maastricht University, P.O. Box 616, 6200 MD Maastricht, The Netherlands. E-mail: pim.peeters@ maastrichtuniversity. $\mathrm{nl}$

$1526-5900 / \$ 36.00$

(C) 2011 by the American Pain Society

doi:10.1016/j.jpain.2011.07.007 social context effects on pain expression. According to the cognitive appraisal model, the degree to which pain is experienced as aversive largely depends on how threatening pain is perceived to be. What the social safety cuing hypothesis adds to this model is the prediction that this threat value of pain can be influenced by the social context, with the context serving as a cue that signals safety or threat. Based on this hypothesis, it would be expected that social threat increases pain expression, caused by an increase in experienced threat of pain. For example, Gray and Wegner ${ }^{11}$ demonstrated that pain stimuli that were perceived as intentionally rather than unintentionally administered were indeed reported to be more painful. However, it remains to be shown that this increase in pain report resulted from an increase in the threat value of the pain.

Another mechanism that could underlie social context effects on pain expression is the communicative goals of the individual in pain. According to communicative approaches, pain expression primarily serves to solicit social support from others. ${ }^{5}$ More specifically, an evolutionary perspective of pain expression ${ }^{25}$ suggests that pain expression is likely to be suppressed when the social context signals threat, such as the presence of an antagonist, and expressing pain may be harmful rather than helpful. In a similar line, the communal coping 
model of pain catastrophizing proposes that specifically individuals who catastrophize about pain use pain expression to solicit social support. ${ }^{20}$ Contrary to the social safety cuing hypothesis, these models predict that social threat reduces pain expression rather than increasing it, since social threat signals a decreased likelihood of social support.

The present study aimed to investigate the influence of a social threat to physical integrity on reported pain intensity and facial expression. The predictions of both the social safety cuing hypothesis and the communicative models of pain expression were compared, by introducing a confederate who intentionally administered less or more pain stimuli to the participant than required. The confederate was present in the same room while administering the pain stimuli, creating a context in which communication was possible. In accordance with the communal coping model, pain catastrophizing was investigated as a possible moderator. Experienced threat of pain was measured to investigate its mediating role proposed by the social safety cuing hypothesis.

\section{Methods}

\section{Ethical Approval}

Approval for the study was granted by the Ethical Committee Psychology from the Maastricht University Faculty of Psychology and Neuroscience.

\section{Design}

A 2 (low/high pain catastrophizing) $\times 3$ (low/neutral/ high social threat) between-subjects design was used in this study. Participants were randomly assigned to 1 of 3 conditions; low social threat (LST), neutral social threat (NST), and high social threat (HST). Furthermore, participants were split into low and high pain catastrophizers around a cut-off score of 16 on the Pain Catastrophizing Scale, the mean score in a previous student population. ${ }^{21}$

\section{Participants}

Eighty-two healthy participants were recruited by spreading flyers throughout the university. Exclusion criteria were chronic pain states, cardiovascular risk factors, and mental disorders. All participants read and signed an informed consent form prior to participating in the experiment.

\section{Electrical Pain Stimuli}

Using a constant current stimulator (Model DS7, Digitimer, Welwyn Garden City, UK), electrocutaneous pain stimuli $(50-\mathrm{Hz}$ block pattern waveforms of 3 seconds duration) were generated at the press of a button. Participants received the stimuli through $2 \mathrm{Ag} / \mathrm{AgCl}$ electrodes spaced at $2 \mathrm{~cm}$, placed just below the inside of the wrist. The function generator was present in the same room as the participants, who were thus able to see that pressing the button initiated a painful stimulus. At the beginning of the experiment, the stimuli were individually calibrated by starting stimulation at $1 \mathrm{~mA}$, and building up with $1 \mathrm{~mA}$ until participants reported a subjective pain intensity of 8 on a scale from 0 to 10 . A suitable level of painful stimulation was reached for all participants, under the safety cut-off point of $20 \mathrm{~mA}$.

\section{Social Threat Manipulation}

The threat value of the social context was experimentally manipulated by varying the perceived willingness of a confederate to inflict pain on the participant. Participants entered the experiment with a confederate (Caucasian female, age 21 years) whom they believed was another participant. They were told that for the purpose of the study, 1 of them would have to administer pain stimuli to the other.

Through a bogus randomization procedure, the confederate would be assigned to administer pain stimuli to the participant. While the confederate administered 5 pain stimuli to the participant in all conditions, the crucial manipulation in this procedure was the way these 5 stimuli were framed. In the NST condition, the participants were told that the confederate was instructed by the experimenter to administer 5 pain stimuli and that no personal choice of the confederate was involved. In the LST condition, participants were told that the confederate was allowed to deliver between 5 and 20 pain stimuli at his own choice, 5 thus being the lowest number allowed. Finally, in the HST condition, participants received the information that the confederate had the choice to administer 1 to 5 pain stimuli, 5 thus being the highest number allowed. An audiotape would prompt the confederate to state out loud the number of stimuli she had chosen in the presence of the participant, while again framing the limits of the condition (for example, in the HST condition: "How many stimuli are you going to administer between 5 and $20^{\prime \prime}$ ?), in response to which the confederate would always answer "5."

\section{Measures}

\section{Demographic Characteristics and Screening}

At the beginning of the experiment, participants were asked to complete a screening questionnaire asking about their gender, age, level of education, and possible exclusion criteria.

\section{Perceived Social Threat}

A social threat questionnaire (STQ) consisted of 12 statements describing aspects of the relation between the participant and the confederate, which were rated on the degree to which participants experienced such a relationship on an 11-point scale from 0 (completely disagree) to 10 (complete agree). To ensure a general measurement of perceived social threat, each item referred to 1 of 3 conceptions of social threat; specific social threat (eg, "I had the feeling the other participant enjoyed hurting me"), social proximity (eg, "I feel close to the other participant"), and social likeability (eg, "the other participant is honest"). 
On the basis of a reliability analysis, 2 items were removed. These 2 items were formulated more negatively than the other items, which may have led to a floor effect of the scores. The remaining scale consisted of 12 items with a high internal reliability (Cronbach's alpha $=.85$ ). In order to check whether the sum score of the STQ reflects 1 construct of social threat, a Principal Factor Analysis of the 12 items was conducted. This analysis yielded a 1 -factor solution that accounted for $46.5 \%$ of the total variance. While the sample size of the present study is too small to consider this result as a reliable validation of the social threat questionnaire, it justifies the use of the STQ sum score as a measure of social threat.

\section{Pain Catastrophizing}

Pain catastrophizing, the extent to which pain is habitually experienced as threatening, was measured with the Dutch version of the Pain Catastrophizing Scale (PCS). ${ }^{19}$ The PCS is a 13-item self-report questionnaire that targets rumination, magnification and feelings of helplessness associated with the experience of pain. The PCS has shown to be a valid and highly reliable instrument (Cronbach's alpha between .85 and $.91^{22}$ ).

\section{Positive and Negative Affect}

Trait positive and negative affect were measured to correct for possible confounding for the effects of pain catastrophizing. Additionally, state positive and negative affect were measured before and after the social threat manipulation to correct for possible confounding for the effects of social threat. Affect measures were taken with the trait and state versions of the Positive and Negative Affect Schedule (PANAS- $t /-\mathrm{s}^{24}$ ) respectively. Both versions of the PANAS contain 10 positive and 10 negative affect items that have to be scored on 5-point Likert scales. Items are summed to obtain positive affect and negative affect scores. While the PANAS-t asks participants to rate the affect items with reference to how they usually feel, the PANAS-s asks the participants to describe how they are feeling at the moment of completing the questionnaire.

\section{Perceived Threat Value of Pain}

The perceived threat value of the pain inflicted during the experimental manipulation was measured with a pain threat questionnaire consisting of 6 items describing threatening pain appraisals, ${ }^{23}$ which the participants rated on the degree to which they agreed to having made that particular threatening appraisal, on an 11point scale from 0 (completely disagree) to 10 (complete agree). Reliability analysis yielded a good internal consistency (Cronbach's alpha $=.91$ ).

\section{Reported Pain Intensity}

Pain intensity was measured by having participants verbally report the intensity of the pain they experienced after each pain stimulus that was administered, by giving it a rating between 0 (no pain at all) and 10 (the most extreme pain) on a numerical rating scale in the form of a thermometer. Verbal report of pain intensity was cho- sen over written report of pain intensity, because verbal report is more likely to be open to both cognitive and communicative influences than written report. This makes for a more fair comparison between the cognitive appraisal and communicative models. All pain reports were averaged for each individual to create a reported pain intensity score.

\section{Facial Pain Expression}

Facial pain expression was coded using an abridged version of the Child Facial Coding System $\left(\mathrm{CFCS}^{3}\right)$. The abridged CFCS consists of the 6 facial actions of the CFCS that are most consistently related to pain expres$\operatorname{sion}^{15,16}$; brow lowering, eye squint, eye squeeze, nose wrinkle, cheek raiser, and upper lip raise. These actions were coded for 3 levels of intensity. The abridged version has shown to be highly comparable with the full CFCS (Peeters, 2010), which has shown good reliability and validity in coding adults' facial pain expressions. $^{23}$

Coding was conducted for each electrocutaneous stimulus that was administered, starting at 1 second before the shock, 3 seconds during the shock, and 2 seconds afterwards, for a total of 6, 1-second coding fragments for each shock. Fragments during which the participant's face was poorly visible because the head was turned, or fragments during which the participant was speaking were coded as invalid. All fragments were coded by a trained coder blind to the experimental condition, who achieved satisfactory interrater reliability according to the formula of Ekman and Friesen ${ }^{8}$ (.70 or higher for each individual action unit) on an extensive, full CFCS training. No interrater reliability was specifically calculated for the present study. An average facial pain expression score was calculated for each individual by dividing the sum of all action units by the number of valid fragments.

\section{Procedure}

At the beginning of the experiment, both the participant and the confederate entered the laboratory together and were seated facing each other at a table. After being informed on the procedures of the experiment, they were asked to complete the demographics and screening questionnaires, after which only participants who reported no exclusion criteria were allowed to continue with the study. Subsequently, both the participants and confederate were asked to read and sign the informed consent form and complete the PANAS-t, PANAS-s, and PCS. Following the questionnaires, the bogus randomization procedure was shown, assigning the participant the role of receiving the pain stimuli administered by the confederate.

At this point, the experimenter brought the confederate to another room to ensure that the calibration of the pain stimulus was done in the most neutral social context possible. The experimenter stayed in the lab to calibrate the electric pain stimulus for the participant. The calibrated intensity of the electric stimuli was maintained for the painful stimuli administered by the confederate. 
After stimulus calibration, the pain threat questionnaire was completed with reference to the most intense stimuli they had just experienced.

Subsequently, the experimenter brought the confederate back to the lab and provided additional instructions to the confederate on how to administer pain stimuli. The experimenter then left the lab and started an audio recording with task instructions. First, information was given to the participant about the range of pain stimuli from which the confederate was requested to choose (LST: 5 to 20, NST: 5, HST: 1 to 5). The confederate always responded to this request by overtly stating that she was going to deliver 5 stimuli. These first instructions were followed by 5 auditory cues signaling the confederate when to deliver the pain stimulus by pressing the button. Nine seconds after each pain stimulus, the participant was cued to verbally report the experienced pain intensity of the stimulus in the presence of the confederate. During this procedure, the participant's face was videotaped to code for facial pain expression.

Following the final pain intensity report, the experimenter returned to the lab and brought the confederate to the other room again. Participants were asked to complete the second pain threat questionnaire, PANAS-s, and the social threat measures. When finished, they were debriefed, thanked, and received a small remuneration for their participation.

\section{Statistical Analyses}

Three statistical analyses were used to test the hypotheses put forward by the communicative and cognitive appraisal models; 2 regression analyses and 1 mediation analysis. First, 2 linear regression analyses were used to investigate the influences of social threat, pain catastrophizing, and their interaction (the variables of interest) on both reported pain intensity and facial pain expression (the dependent variables). Several covariates were entered to control for various other influences on reported pain intensity and facial pain expression.

Both regression analyses used a forward strategy, with reported pain and experienced threat of pain at calibration entered as covariates in the first step, in order to correct for small baseline differences in electrocutaneous stimulus perception still present after calibration. Next, trait negative and positive affect, and change in state positive and negative affect, were entered in order to correct for any confounding effects of mood for the social context manipulation. Finally, pain catastrophizing, the social threat measure, and their interaction were entered as the independent variables of interest. To investigate whether any social threat effects were mediated by the experienced threat value of the pain, the last analysis consisted of a mediation analyses conducted according to the steps described by Baron and Kenny. ${ }^{1}$

All variables were centered for the purpose of these analyses. In the case of a significant interaction effect, the simple effects of social threat per level of pain catastrophizing were investigated. For these simple effect analyses, pain catastrophizing was dichotomized around a cut-off score of 16, the mean score for the PCS' Dutch version in a previous student population ${ }^{21}$, and dummy coded with the lowest scoring group being the constant. Variable Inflation Factors for all the analyses did not exceed 2, indicating that there was no problem of multicollinearity.

Initially, the intention was to use the 3 separate social context conditions as independent variables. However, a manipulation check indicated no significant differences between the LST and NST conditions, mostly due to the large spread in scores within all conditions. Leaving out the NST condition resulted in too small a sample size for reliable analyses; hence, alternatively to using the conditions, the individual scores on the social threat measure were used as the independent variable in the analyses. While this approach had no noticeable effects on the effect sizes of social threat, pain catastrophizing, and their interaction as compared with an analysis with the LST and HST conditions, it increased statistical power sufficiently to conduct reliable analyses.

\section{Results}

\section{Participant Descriptives}

A total sample of 82 participants completed the study. Thirteen participants were excluded for analyses because they expressed suspicion about the role of the confederate. Data of another 2 participants had to be excluded from the study because no pain report or facial pain expression data were available, due to technical problems with videotaping. The total sample that was included into the analyses contained 67 participants, 49 women and 18 men (Tables 1 and 2).

\section{Reported Pain Intensity}

Regression analyses on the reported pain intensity yielded a statistically significant social threat $\times$ pain catastrophizing interaction $(B=-.252 ; P<.05$ : Fig 1 and Table 3). Analyses of the simple effects showed that increased social threat only significantly increased pain ratings for high pain catastrophizers (STQ: $B=-.461 ; P<$ .05) and not for low pain catastrophizers (STQ: $B=$ $.013 ; P=.955)$.

\section{Facial Pain Expression}

Regression analyses on the facial pain expression with the total score on the social threat questionnaire, pain catastrophizing, and their interaction as independent variables yielded a significant main effect for social threat ( $B=.359 ; P<.01$; Fig 2 and Table 3$)$, with increased social threat leading to reduced facial expression. No significant interaction effect $(B=.079 ; P=.550)$ or main effect for pain catastrophizing $(B=.012 ; P=.935)$ was found.

\section{Social Cuing Hypothesis}

To examine whether the social context influences may have been mediated by the perceived threat value of the pain, mediation analyses were conducted. Having already produced the total effect $(c+a * b)$ in the previous 
Table 1. Means and Standard Deviations on the Study's Most Important Measures

\begin{tabular}{lcccc}
\hline & TotAL & LST CoNDITION & NST CoNDITION & HST CONDITION \\
& MEAN (SD) & MEAN (SD) & $15.42(8.22)$ & $14.50(8.56)$ \\
\hline Pain catastrophizing & $15.12(7.97)$ & $15.43(7.36)$ & $7.83(9.65)$ & $10.29(9.12)$ \\
Pain threat premeasure & $10.05(10.64)$ & $12.33(12.89)$ & $6.29(9.70)$ & $8.05(8.80)$ \\
Pain threat postmeasure & $8.00(10.02)$ & $9.90(11.61)$ & $58.75(11.26)$ & $36.27(15.58)$ \\
Social threat questionnaire & $53.17(18.06)$ & $64.50(13.76)$ & $6.67(1.40)$ & $6.82(1.32)$ \\
Pain rating & $6.61(1.37)$ & $6.32(1.41)$ & $.70(.94)$ & $.23(.43)$ \\
Facial pain expression & $.57(.81)$ & $.78(.88)$ & & MEAN \\
\hline
\end{tabular}

Abbreviations: LST, low social threat; NST, neutral social threat; HST, high social threat.

analyses, the second step of the mediation analyses (path a) ${ }^{1}$ included the same independent variables but now with perceived threat of the pain (after the pain task) as dependent variable. These analyses yielded no significant effects for the social threat measure $(B=-.003$; $P=.956)$ or interaction between the social threat measure $\times$ pain catastrophizing $(B=-.055 ; P=.368)$, demonstrating that the experienced threat of pain was not influenced by social threat, and hence did not mediate the effects of social threat on reported pain intensity or facial pain expression.

\section{Discussion}

The present study investigated the influence of social threat on reported pain intensity and facial expression of pain. Intriguingly, social threat was found to influence reported pain intensity and facial expression in opposite manners, increasing the pain report of high pain catastrophizers, but at the same time decreasing the facial pain expression of all participants. These results are notable as they point to particular conditions in which the report of pain and the nonverbal expression of pain are dissociated in the same individuals at 1 single instance. So far, no such findings have been reported.

While social threat had no influence on the pain report of low pain catastrophizers, high pain catastrophizers reported increased pain when experiencing high social threat than when experiencing low social threat. These results resemble the findings of Gray and Wegner, ${ }^{11}$ demonstrating that pain that is intentionally caused by another person hurts more than when occurring unintentionally, with the exception that the effects of social threat appear to be specific to high pain catastrophizers, a factor that was not included in their study. Pain catastrophizing has repeatedly shown to be a potent predictor of self-reported pain ${ }^{20}$ and the current study demonstrates that these effects not only generalize towards a socially threatening situation, but also increase under social threat. Furthermore, as both trait affect and any changes in state affect brought about by the social threat manipulation were controlled for in the present study, social threat appears to have a unique effect on reported pain over and beyond any effects it may already have through mood changes. While the present study did not include a threat manipulation, it is possible that only high pain catastrophizers experienced the pain to be threatening enough to be influenced by the social threat cue (Pearson $r=.46$ between pain catastrophizing and experienced threat of pain). Nevertheless, no support was found for the assumption from the safety cue hypothesis that the association between social threat and pain report is mediated by the experienced threat value of pain.

It can be hypothesized that rather than affecting the experienced threat of the pain, social threat as operationalized in the current study causes a different aspect of pain to become more aversive. For example, being intentionally hurt by another person may increase the unpleasantness of pain through its perceived social unfairness. Social influences on pain are unlikely to be limited to a single aspect of the pain experience or expression. It is likely that some social influences act through affective and cognitive mechanisms such as experienced threat, helplessness, or attention, while others may act through more social mechanisms including perceived justice or social exclusion. ${ }^{6,7}$ The social threat effect on reported pain intensity found in this study is more likely to belong to the latter category. An even more elaborate investigation of different aspects of pain than the present study would be necessary to test this hypothesis.

Contrary to the results for reported pain intensity, social threat was found to decrease facial pain expression,

Table 2. Correlations Between the Study's Most Important Measures

\begin{tabular}{|c|c|c|c|c|c|}
\hline & $\begin{array}{l}\text { PAIN THREAT } \\
\text { PrEMEASURE }\end{array}$ & $\begin{array}{c}\text { PAIN } \\
\text { THREAT POSTMEASURE }\end{array}$ & $\begin{array}{l}\text { SOCIAL THREAT } \\
\text { QUESTIONNAIRE }\end{array}$ & $\begin{array}{c}\text { PAIN } \\
\text { RATING }\end{array}$ & $\begin{array}{l}\text { FACIAL PAIN } \\
\text { EXPRESSION }\end{array}$ \\
\hline Pain catastrophizing & .476 & .458 & -.107 & .222 & .124 \\
\hline Pain threat premeasure & & .915 & -.177 & .365 & .238 \\
\hline Pain threat postmeasure & & & -.169 & .352 & .224 \\
\hline Social threat questionnaire & & & & -.225 & .235 \\
\hline Pain rating & & & & & .236 \\
\hline
\end{tabular}




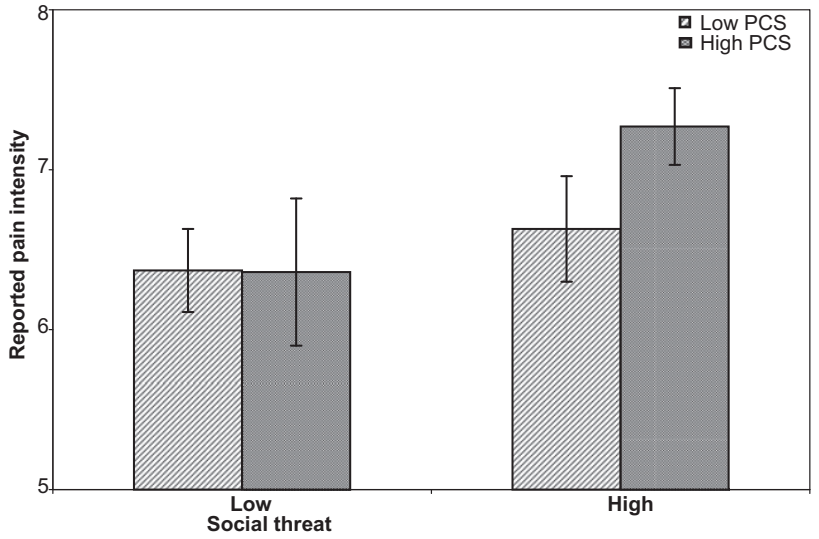

Figure 1. Reported pain intensity by pain catastrophizing and social threat based on the social threat questionnaire. Pain catastrophizing was dichotomized around a score of 16 , while social threat was dichotomized around its median.

irrespective of the level of pain catastrophizing, which is in line with predictions from the evolutionary perspective expressed by Williams. ${ }^{25}$ According to such a perspective, ${ }^{10}$ facial pain expression, like any other expression of emotion, serves a primarily communicative function, aiming to solicit help from others. While in-group individuals, like kin, may be motivated to help an individual who expresses pain, same species competitors who wish the individual ill, may understand that a signal to solicit help also signals that the individual is less able to defend himself, providing an ideal opportunity to aggress. Hence, expression of pain would be more dangerous when a situation is socially threatening, causing individuals to express less pain.

While the findings support a communicative function of facial pain expression, they do not support the claim of the communal coping model, ${ }^{20}$ that high pain catastrophizing people display pain expression in a functionally different way than low pain catastrophizers. However, the lack of an effect for pain catastrophizing on facial expression may also be due to a procedural artifact. Pain stimuli were calibrated for each participant

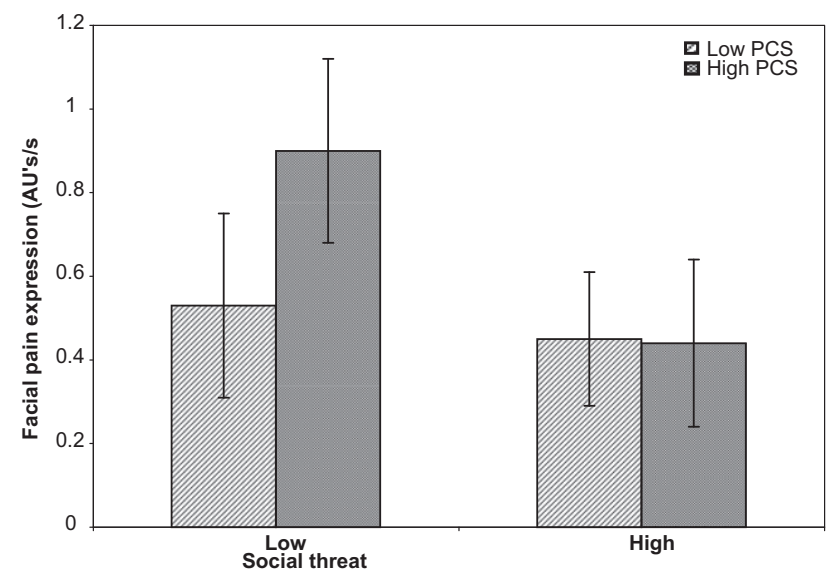

Figure 2. Facial pain expression by pain catastrophizing and social threat based on the social threat questionnaire. Pain catastrophizing was dichotomized around a score of 16 , while social threat was dichotomized around its median.
Table 3. Regression Coefficients for the Analyses on Reported Pain and Facial Pain Expression

\begin{tabular}{|c|c|c|c|c|c|c|}
\hline \multirow[b]{2}{*}{ VARIABLES } & \multicolumn{3}{|c|}{ REPORTED PAIN } & \multicolumn{3}{|c|}{$\begin{array}{l}\text { FACIAL PAIN } \\
\text { EXPRESSION }\end{array}$} \\
\hline & $\beta$ & $t$ & SIG. & $\beta$ & $t$ & SIG. \\
\hline $\begin{array}{l}\text { Baseline pain after } \\
\text { calibration }\end{array}$ & .273 & 2.483 & $.016^{*}$ & -.149 & -1.159 & .251 \\
\hline Trait positive mood & -.134 & -1.198 & .236 & .091 & .742 & .461 \\
\hline Trait negative mood & -.329 & -3.034 & $.004^{*}$ & -.008 & -.061 & .952 \\
\hline $\begin{array}{l}\text { Positive mood } \\
\text { change }\end{array}$ & -.059 & -.503 & .617 & .184 & 1.473 & .146 \\
\hline $\begin{array}{l}\text { Negative mood } \\
\text { change }\end{array}$ & .236 & 2.018 & $.048^{*}$ & .184 & 1.473 & .146 \\
\hline Pain catastrophizing & .214 & .130 & .897 & -.009 & -.064 & .949 \\
\hline Social threat & -.165 & -1.496 & .140 & .359 & 2.817 & $.007^{*}$ \\
\hline $\begin{array}{l}\text { Social threat } \times \text { pain } \\
\text { catastrophizing }\end{array}$ & -.252 & -2.036 & $.046^{*}$ & - & - & - \\
\hline
\end{tabular}

* Significant at $P=.05$

individually, and as baseline experience of threat was controlled for, individual differences in pain sensitivity may have been minimized at the start of the study. Any difference in facial expression between high and low pain catastrophizers in other studies may have been caused by a difference in the experience of the pain stimuli, ${ }^{17}$ which causes the communication goals of high pain catastrophizers to be focused more strongly on soliciting support versus protecting oneself from social threats.

The opposite effects of social threat on reported pain intensity and facial pain expression suggest different functions for both expressions of pain. While others have noted that both forms of pain expression are likely to be driven by different mechanisms for encoding pain experience ${ }^{13}$ and represent different adaptive functions, ${ }^{26}$ to the knowledge of the authors the present study is the first to demonstrate a single manipulation to have opposite effects on reported intensity an facial expression. These findings underscore the necessity of studying the distinct mechanisms behind reported intensity and facial expression, and the conditions under which both forms of pain expression are likely to covary or diverge. The latter is especially important with an eye on pain assessment methods that are based (often by necessity) on only 1 form of pain expression, such as pain assessment based on facial pain expression in neonates ${ }^{12}$ or cognitively impaired adults or elderly. ${ }^{4}$ To estimate an individual's pain experiences, observers decode the pain expression which in turn arises from the individual encoding of the pain experience. ${ }^{13}$ More detailed knowledge about how these encoding processes of pain expression are influenced by environmental factors may help in the development of more sensitive pain measures.

The findings of the present study encourage further research on the effects of social threat cues on pain. It would be intriguing to investigate other kinds of social threat cues, such as in-group versus out-group differences, ${ }^{14}$ experienced social discrimination, and the 
withdrawal of preexisting social support as another form of threat, or excessive performance demands by an authority figure. Another interesting avenue of research would be to investigate other kinds of moderators of social threat effects, such as gender, sociotropy/autonomy

\section{References}

1. Baron RM, Kenny DA: The moderator-mediator variable distinction in social psychological research: Conceptual, strategic and statistical considerations. J Pers Soc Psychol 51: $1173-1182,1986$

2. Brown JL, Sheffield S, Leary MR, Robinson ME: Social support and experimental pain. Psychosom Med 65:276-283, 2003

3. Chambers CT, McGrath PJ, Gilbert CA, Craig KD: Child Facial Coding System - revised manual. Halifax, NS, IWK-Grace Health Centre, Dalhousie University and University of British Columbia, 1996

4. Cohen-Mansfield J: The relationship between different pain assassments in dementia. Alzheimer Dis Assoc Disord 22:86-93, 2008

5. Craig KD: The social communication model of pain. Can Psychol 50:22-32, 2009

6. DeWall CN, Baumeister RF: Alone but feeling no pain: Effects of social exclusion on physical pain tolerance and pain threshold, affective forecasting and interpersonal empathy. J Pers Soc Psychol 91:1-15, 2006

7. Eisenberger NI, Lieberman MD, Williams KD: Does rejection hurt? An fMRI study of social exclusion. Science 302: 290-292, 2006

8. Ekman P, Friesen W: Investigator's Guide to the Facial Action Coding System. Palo Alto, CA, Consulting Psychologists Press, 1978

9. Flor $\mathrm{H}$, Breitenstein $\mathrm{C}$, Birbaumer N, Fürst $\mathrm{M}$ : A psychophysiological analysis of spouse solicitousness towards pain behaviors, spouse interaction, and pain perception. Behav Ther 26:255-272, 1995

10. Fridlund A: Evolution and facial action in reflex, social motive, and paralanguage. Biol Psychol 32:3-100, 1991

11. Gray K, Wegner DM: The sting of intentional pain. Psychol Sci 19:1260-1262, 2008

12. Grunau RE, Oberlander T, Holsti L, Whitfield MF: Bedside application of the Neonatal Facial Coding System in pain assessment of premature infants. Pain 76:277-286, 1998

13. Hadjistavropoulos T, Craig KD: A theoretical framework for understanding self-report and observational measures of pain: A communications model. Behav Res Ther 20: 551-570, 2002 preferences, or sensitivity to rejection. By extending the research on social threat effects, their moderators, and working mechanisms, a better understanding of various forms of pain expression may be gained in relation to the social context in which they are displayed.

14. Platow MJ, Voudouris NJ, Coulson M, Gilford N Jamieson R, Najdovski L, Papaleo N, Pollard C, Terry L: Ingroup reassurance in a pain setting produces lower levels of physiological arousal: Direct support for a selfcategorization analysis of social influence. Eur J Soc Psychol 37:649-660, 2007

15. Prkachin KM: The consistency of facial expressions of pain: A comparison across modalities. Pain 51:297-306, 1992

16. Prkachin KM, Craig KD: Expressing pain: The communication and interpretation of facial pain signals. J Nonverbal Behav 19:191-205, 1995

17. Severeijns $R$, Vlaeyen JWS, van den Hout MA: Do we need a communal coping model of pain catastrophizing? An alternative explanation. Pain 111:226-229, 2004

18. Sullivan MJL, Adams H, Sullivan ME: Communicative dimensions of pain catastrophizing: Social cueing effects on pain behaviour and coping. Pain 107:220-226, 2004

19. Sullivan MJL, Bishop S, Pivik J: The pain catastrophizing scale: Development and validation. Psychol Assess 7:52-64, 1995

20. Sullivan MJL, Thorn B, Haythornthwaite JA, Keefe F, Martin M, Bradley LA, Lefebvre JC: Theoretical perspectives on the relation between catastrophizing and pain. Clin J Pain 17:52-64, 2001

21. Van Damme S, Crombez G, Bijttebier P, Goubert L, Van Houdenhove B: A confirmatory factor analysis of the Pain Catastrophizing Scale: Invariant factor structure across clinical and non-clinical populations. Pain 96: 319-324, 2002

22. Van Damme S, Crombez G, Vlaeyen JWS, Goubert L, Van den Broeck A, Van Houdenhove B: De Pain Catastrophizing Scale: Psychometrische karakteristieken en normering. Gedragstherapie 3:211-222, 2000

23. Vlaeyen JWS, Hanssen MMP, Dautzenberg A Peters ML, Goubert L, Sullivan ML, Morley S: Threat of pain influences social context effects on verbal pain report and facial expression. Behaviour Research and Therapy 47: 774-782, 2009

24. Watson D, Clark LA, Tellegen A: Development and validation of brief measures of Positive and Negative Affect: The PANAS Scales. J Pers Soc Psychol 54:1063-1070, 1988

25. Williams ACdC: Facial expression of pain: An evolutionary account. Behav Brain Sci 25:439-488, 2002

26. Williams ACdC, Craig KD: A science of pain expression? Pain 125:202-203, 2006 